\title{
Fine needle aspiration cytology and the choice of fixatives in the diagnosis of tuberculous adenitis at Muhimbili National Hospital, Dar-es-Salaam, Tanzania
}

\author{
HENRY A. MWAKYOMA*, GRACE S. MALIVA and SAYOKI G. MFINANGA \\ Muhimbili University of Health and Allied Sciences, P.O. Box 65001, Dar es Salaam, Tanzania \\ Muhimbili School of Medical Laboratory Sciences, Dar es Salaam, Tanzania \\ National Institute for Medical Research, Muhimbili Research Centre, Dar es Salaam, Tanzania
}

\begin{abstract}
Tuberculosis (TB) is a global public health problem. Despite recent success in improving TB cure rates, diagnosis and case detection remains a major obstacle to its control. In this study the usefulness of four fixatives in improving Acid Fast Bacilli (AFB) microscopy diagnosis of TB was assessed. The study was conducted at Muhimbili National Hospital in Dar es Salaam, Tanzania. A total of 560 cases of peripheral lymphadenopathies had fine needle aspiration cytology (FNAC) done. Out of these cases, $110(19.6 \%)$ were diagnosed by ZN stain to be TB lymphadenitis. Different types of fixatives in diagnosis of TB were compared. Each specimen was smeared and fixed on to four glass slides using four fixatives (95\% ethyl alcohol, Acetone, Carnoy's fluid and 10\% neutral buffered formalin), and one slide fixed using air dried method. Subsequently, Zeelh-Neelsen (ZN) stained slides were examined under a microscope for AFB. Of the 101 cases, 53 were TB adenitis, as diagnosed by AFB smear microscopy on ZN stained slides, and 48 were adenitis due to other causes. The diagnostic yield of AFB was highest when using $95 \%$ ethyl alcohol which was observed to be $30.2 \%(16 / 53)$ when AFB was $1+, 58.5 \%(31 / 53)$ when AFB was $2+$ and $11.3 \%(6 / 53)$ when AFB was 3+, and making a total yield of $100 \%$ (53/53). Air dried smears also gave a relatively high yield with total yield of $94.3 \%$ (50/53). When $95 \%$ ethyl alcohol was used as a gold standard fixative, air dried slides had highest sensitivity (94.3\%) and all fixatives had high specificity. The study has demonstrated that $95 \%$ ethyl alcohol is an excellent fixative. It is therefore recommended that $95 \%$ ethyl alcohol be used to form part of the routine fixation of FNAC smears.
\end{abstract}

Keywords: tuberculosis, diagnosis, fixatives, FNAC smears, Tanzania

\section{Introduction}

Tuberculosis (TB) is a major public health problem worldwide (WHO, 2008). Tanzania is among the 22 countries with the highest burden of tuberculosis in the world. In 2006, about 62,100 tuberculosis (TB) cases were reported, of which smear positives, negatives, relapses and extra-pulmonary cases were 39.8\%,32.4\%, 2.9\%, and 20.3\%, respectively (Ministry of Health, unpubl.). The notification rate of smear positive tuberculosis rose from about $20 / 100,000$ in 1979 to $63 / 100,000$ in 2006. The proportion of smear negative and extrapulmonary cases rose from $20 \%$ and $11 \%$ in 1979 to $32 \%$ and $20 \%$ in 2006 . The extrapulmonary tuberculosis (EPTB) has increased almost 10-fold in the same period from 1232 to 12,621 cases (Ministry of Health, unpubl.).

Lymphadenitis is one of the common manifestations of extrapulmonary form of tuberculosis (Kishore Reddy et al., 2008). In a study by Mwakyoma et al. (1996) at the Muhimbili National Hospital in Tanzania, TB adenitis was found to account for $46 \%$ of all conventional lymphnode biopsies performed. TB lymphadenitis has been found to represent about $20 \%$ of all peripheral lymphadenopathies at the Department of Histopathology and

\footnotetext{
* Correspondence: Henry A. Mwakyoma; E-mail: hmwakyoma@yahoo.com
} 
Morbid Anatomy of the MNH in 2002. In India, tuberculous lymphadenitis was found to comprise about half of all cases of extrapulmonary TB in 2005 (Fraser et al., as cited by Kishore Reddy et al., 2008). Cases of TB lymphadenopathies are on the increase as extrapulmonary manifestation in HIV individuals (Gita \& Chew, 2000; Kishore Reddy et al., 2008).

Despite recent success in improving cure rates for tuberculosis, diagnosis and case detection remains a major obstacle to its control (Pai et al., 2009). This is because, in many developing countries, the diagnosis of tuberculosis continues to be surrounded by considerable uncertainty (Getachew \& Tesfahugne, 1999; Hooper, 1972; Bezabin et al., 2002). In Tanzania, the diagnosis of tuberculosis is seldom confirmed by culture. It usually relies on a constellation of symptoms, clinical signs, radiological findings and use of smear microscopy for Acid fast bacilli (AFB).

The role of fine needle aspirate cytology (FNAC) in the evaluation of lymphadenopathies is well known (Das, 1991; Reid et al., 1998; Kline et al., 2000; Gita \& Chew, 2000). When compared with conventional lymph node biopsy, the diagnostic yield of FNAC gives a better sensitivity and specificity as an initial procedure in the diagnosis of lymphadenopathy (Sunpaweravong et al., 1999; Kishore Reddy et al., 2009). When compared with conventional lymph node biopsy, FNAC procedure is simple, non-traumatic and does not require the use of anaesthesia and can help clinicians as an initial investigation for the diagnosis of lymphadenopathy (Bezabih et al., 2002; Kishore Reddy et al., 2009). At the Muhimbili National Hospital in Dar es Salaam, Tanzania, it has been observed that using FNAC smears there is more diagnostic yield of acid fast bacilli when the smear is air-dried than when is put in a fixative (H. Mwakyoma et al., unpubl.). This study was therefore undertaken to assess the usefulness of FNAC and diagnostic yield of tubercle bacilli by using different methods and types of fixatives in patients with TB lymphadenitis.

\section{Materials and Methods}

\section{Study design, subjects and procedures}

This study was carried out at Muhimbili National Hospital (MNH) in Dar es Salaam, Tanzania. MNH is a referral and university teaching hospital with a 1500 bed facility, attending 1,000 to 1,200 outpatients week, admitting 1,000 to 1,200 inpatients per day (http://www.mnh.or.tz/). In this prospective cross-sectional study, all patients presenting with enlarged peripheral lymphnodes attending the outpatient FNAC clinic at the Department of Histopathology and Morbid Anatomy, from September 2006 to February 2007 were enrolled.

A total of 560 cases of peripheral lymphadenopathies had FNAC done. Out of these cases, $110(19.6 \%)$ were diagnosed by ZN stain to be TB lymphadenitis. FNAC materials from 101 cases were used to assess usefulness of different types of fixatives in diagnosis of TB. Of the 101 cases, 53 were TB adenitis, as diagnosed by Acid Fast Bacilli (AFB) smear microscopy on ZN stained slides, and 48 were adenitis due to other causes. Neither the investigator nor the technician knew which materials were from tuberculous adenitis cases and which were from non- tuberculous adenitis cases.

FNAC was performed using a 22 gauge needle attached to a $10 \mathrm{ml}$ plastic syringe, mounted to a syringe holder for a single hand grip. The aspirated material was deposited onto five clean glass slides. One slide was air-dried while others were immersed in different types of fixatives namely: 95\% ethyl alcohol, Acetone, Carnoy's fluid and 10\% neutral buffered formalin. Slides 
were then fixed at varying duration of fixation as follows: Acetone for 15 minutes, $95 \%$ Ethyl alcohol for 20 minutes, 10\% neutral buffered formalin for 30 minutes and Carnoy's fluid for 15 minutes. After fixation, the slides were stained, using the Zeehl-Neelsen (ZN) method, in which the following procedure was used: The slides were brought down to water and then flooded with basic carbofuchsin. Heat was then applied gently until steam rose up, and then left the slides to stain for 10 minutes. The slides were thereafter rinsed in water, decolourised with $1 \%$ acid alcohol until no stain come out, and again rinsed in water and subsequently counterstained with methylene blue for 1 minute. The slides were then blot-dried and dehydrated in 3 changes of absolute alcohol. Lastly slides were cleared in xylene, mounted in DPX and left to dry. The slides were then examined under a light microscope (Olympus C x 31- Japan) and results were reported according to the World Health Organization and International Union Against Tuberculosis and Lung Disease procedure (WHO, 2000). Sputum smears from known and proven TB cases were used as positive controls for internal quality control.

\section{Data analysis}

A formatted computer Excel software, using standard definition for sensitivity and specificity (Ott, 1988) was used to calculate sensitivity and specificity to yield tubercle bacilli (AFB) for each fixative. A 95\% ethyl-alcohol was used as a gold standard to give additional information on the sensitivity and specificity of others fixatives because its yield for AFB gave $100 \%$ positivity in all 53 cases of TB adenitis.

\section{Results}

Of the 101 patients, 46 (45.5\%) were males and 55 (54.5\%) were females. The mean age was 29.5 years (range $=7$ months to 76 years). Fifty-three (52.5\%) patients had tuberculous adenitis while 48 $(47.5 \%)$ had non-tuberculous lymph node enlargement. Of the 53 patients with TB adenitis, 25 $(47.2 \%)$ were males while 28 (52.8\%) were females.

Table 1: The positivity pattern on the yield of tubercle bacilli according to the type of fixative

\begin{tabular}{|c|c|c|c|c|}
\hline \multirow[t]{2}{*}{ Type of fixative } & \multirow[b]{2}{*}{+} & \multicolumn{2}{|c|}{ Yield of TB bacilli } & \multirow[t]{2}{*}{ Total } \\
\hline & & ++ & ++ & \\
\hline Acetone & 5 & 0 & 0 & 5 \\
\hline 95\% ethyl alcohol & 16 & 31 & 6 & 53 \\
\hline Carnoy's fluid & 11 & 0 & 0 & 11 \\
\hline $10 \%$ neutral buffered formalin & 23 & 5 & 0 & 28 \\
\hline Air dried (no fixation) & 29 & 16 & 5 & 50 \\
\hline
\end{tabular}

The diagnostic yield of tubercle bacilli was highest when using 95\% ethyl alcohol which was observed to be $30.2 \%(16 / 53)$ when AFB was $1+, 58.5 \%$ (31/53) when AFB was 2+, and $11.3 \%(6 / 53)$ when AFB was 3+, and therefore making a total yield of $100 \%(53 / 53)$. The yield was less in other fixatives namely: Acetone, Carnoy's and 10\% neutral buffered formalin. Air dried smears also gave a relatively high yield with total yield of $94.3 \%$ (50/53). When used $95 \%$ ethyl alcohol as golden standard fixative, air dried fixative had the highest sensitivity (94.3\%). All fixative appeared to have at least more 95\% specificity. All 53 cases were diagnosed by slides fixed in 95\% ethyl alcohol, followed by neutral 10\% buffered formalin (28 cases), Carnoy's fluid (11 cases) and acetone (5 cases). However, slides which were air-dried gave a total of 50 cases of positive TB cases, very close to that of $95 \%$ ethyl alcohol (Table 1 ). 
The sensitivity and specificity pattern of fixatives when compared to $95 \%$ ethyl-alcohol, the air dried slides gave the highest sensitivity to AFB (94.3\%), followed by $10 \%$ neutral buffered formalin (52.8\%), Carnoy's fluid (20.8\%), and acetone (9.6\%). However, all fixative including the air-dried slides had specificity of $100 \%$ (Table 2 ).

Table 2: Results of Zeelh-Neelsen staining as related to types of fixatives

\begin{tabular}{llll}
\hline Type of fixative & TB +ve cases & TB -ve cases & Sensitivity (\%) \\
& $\mathbf{N}=\mathbf{5 3}$ & $\mathbf{N = 4 8}$ & \\
\hline Acetone & 5 & 95 & 9.6 \\
Carnoy's fluid & 11 & 90 & 20.8 \\
10\% neutral buffered formalin & 28 & 73 & 52.8 \\
Air dried & 50 & 51 & 94.3 \\
\hline
\end{tabular}

\section{Discussion}

Tuberculous adenitis is one of the most common causes of lymph node enlargement. In this study, about half of lymphadenopathies were tuberculous in nature. This is in agreement with other studies done in Tanzania and elsewhere (Mwakyoma et al., 1996; Bezabin et al., 2002; Kishore Reddy et al., 2008). The slightly higher rate observed in this study is likely to be related to the use different fixatives in the fixation of the smears. The use of $95 \%$ ethyl alcohol in this study as a fixative resulted into a very high pick-up rate of tubercle bacilli. Relatively low pick-up rates have been reported in other studies elsewhere (Singh et al., 1989; Gita \& Chew, 2000).

The study has also revealed that the majority of patients who had TB adenitis were young individuals. This finding agrees with studies done in Tanzania and elsewhere (Mwakyoma et al., 1996; Reid et al., 1998; Zeer et al., 2000). The proportion females with TB lymphadenitis were slightly higher than males. A number of previous studies in Tanzania and elsewhere indicate that males and equally affected (Mwakyoma et al., 1996; Sunpaweravong et al., 1999).

Different types of fixatives were employed in the fixation of FNAC smears in order to determine their diagnostic yield of tubercle bacilli. The 95\% ethyl alcohol yielded the highest number of tubercle bacilli and gave the highest sensitivity as compared to acetone, Carnoy's and formalin. The reason as to why fixatives employed in our study gave different yield of tubercle bacilli after ZN staining procedure on smears could not easily be elucidated. However, this is likely to be related to their differences in chemical properties, their interaction with constituents of the mycobacterium cell wall and the dye, carbolfuchsin. It is known that ethyl alcohol alters the structure of proteins primarily by disruption of hydrophobic bonds which contribute to maintenance of the tertiary structure of proteins (Kiyoshi, 1976; Ellis \& Zabrowany, 1993). Hydrogen bonds appear to be more stable in ethanol than in water and thus affecting tertiary structure of proteins and preserving their secondary structure (Bancroft \& Stevens, 1996; Leong \& James, 1996; Noguchi et al., 1997 ).

Smears which were air dried also gave a high sensitivity for AFB. In this case the components of mycobacterium were not altered because there was no prior fixation. Heating is required for the stain penetration as it softens the high lipid content of the cell wall (mycolic acid and wax D (Bancroft \& Stevens, 1996; Leong \& James, 1996; Noguchi et al., 1997).

This study had its limitations in that we did not perform PCR as a diagnostic method which could be more sensitive in the diagnosis of tubercle bacilli in the smears from FNAC and therefore could have be used as gold standard. However, PCR is an expensive method to use in hospitals as a routine method especially in developing countries like Tanzania. 
In conclusion, the study has demonstrated that different types of fixatives when applied on FNAC smears from patients with enlarged lymph node due to TB adenitis have different yield and sensitivity to tubercle bacilli. The 95\% ethyl alcohol gave the highest yield, while air dried fixative had highest sensitivity to tubercle bacilli in FNAC smears to patients with TB adenitis. The findings confirm earlier observations that $95 \%$ ethyl alcohol is an excellent fixative to use in fine needle aspiration smears from patients suspected to have TB adenitis (Saluja \& Ajinyka, 2000; Bezabih et al., 2002; Hirachand et al., 2009).The findings also indicate that air-dried smears may also be used as a second option where 95\% ethyl-alcohol is not available. FNAC has the advantage of being a reliable diagnostic tool as it may help in averting the more invasive surgical procedures undertaken in the diagnosis of tuberculous adenitis (Bezabih et al. 2002). Furthermore, it is recommended to reserve some slides for a smear to be fixed in $95 \%$ ethyl alcohol for subsequent use in $\mathrm{ZN}$ staining procedure on routine basis, especially in this era of HIV/AIDS where the prevalence of extrapulmonary tuberculosis is on the increase.

\section{Acknowledgements}

We would like to thank Mr. T. Chonde of National Institute of Medical Research, Muhimbili Centre, for his technical and logistic assistance.

Received 25 November 2009

Revised 28 May 2010

Accepted 1 June 2010

\section{References}

Bancroft, J.D. \& Stevens, A. (1996) Theory and Practice of Histological Technique pp 766, Churchl Livingstone, Edinburgh.

Bezabin, M., Mariam, D.W. \& Selassie, S.G. (2002) Fine needle aspiration of suspected tuberculous lymphadenitis. Cytopathology 13, 284-290.

Das, D.K. (1991) Lymphnodes. In: Comprehensive Cytology. M Bibbo (ed.). Philadelphia WB Saunders, pp. 671-702.

Ellis, L.A. \& Zabrowany, J. (1993) Safer staining method for acid fast bacilli. Journal of Clinical Pathology 46, 559-560.

Getachew, A. \& Tesfahugne, Z. (1999) Is fine needle aspiration cytology a useful tool for the diagnosis of tuberculous lymphadenitis? East African Medical Journal 76, 260-263.

Gita, J. \& Chew, M.C. (2000) Fine needle aspiration cytology of lymphnodes in HIV-infected individuals. Acta Cytologica 44, 60-66.

Hirachand, S., Lakhey, M., Akhter, J. \& Thapa, B. (2009) Evaluation of fine needle aspiration cytology of lymph node in Kathmandu Medical College, Teaching Hospital. Kathmandu University Medical Journal 7, 139-142.

Hooper, A.A. (1972) Tuberculous peripheral lymphadenitis. British Journal of Surgery 59, 353.

Kishore Reddy, V.C., Asparna, S., Prasad, C.E., Srinivas, A., Triveni, B., Gokhale, S. \& Krishna Moorthy, K.V. (2008) Mycobacterial culture of fine needle aspirate - a useful tool in diagnosing tuberculous lymphadenitis. Indian Journal of Medical Microbiology 26, 259-261. 
Kiyoshi, H. (1976) The nature of Mycobacterium acid-fastness. Biotechnic and Histochemistry 5, 255-260.

Kline, T.S., Kannan, V. \& Kline, I.K. (2000) Lymphadenopathy and aspiration biopsy cytology: review of 376 superficial nodes. Cancer lymphnodes HIV-infected individuals. Acta Cytologica 44, 60-66.

Leong, A.S.Y. \& James, C.L. (1996) A Handbook of Surgical Pathology. Newyork Churchil Livingstone, $321 \mathrm{pp}$.

Mwakyoma, H.A., Mbembati, N.A.A., Aboud, M.M. \& Kahamba, J. (1996) The diagnostic value of lymphadenopathies at Muhimbili Medical Centre, Dar es Salaam: a retrospective study. Tanzania Medical Journal 11, 4-8.

Noguchi, M., Furuya, S., Takeuchi, T. \& Hirohashi, S. (1997) Modified formalin and Methanol fixation methods for molecular biological and morphological analyses. Pathology International 47, 685-691.

Ott, L. (1988) Statistics: An Introduction to Statistical Methods and Data Analysis. $3^{\text {rd }}$ Boston, MA: PWS-KENT Publishing Co.

Pai, M., Minion, J., Sohn, H., Zwerling, A. \& Perkins, M.D. (2009) Novel and improved technologies for tuberculosis diagnosis: progress and challenges. Clinical Chest Medicine 30, 701-716.

Reid, A.H., Milled, R.F. \& Kogan, G.I. (1998) Diagnostic utility of fine needle aspiration cytology in HIV-infected patients with lymphadenopathy. Cytopathology 9, 230-239.

Saluja, J.G. \& Ajinyka, M.S. (2000) Comparative study of fine needle aspiration cytology, histopathology and bacteriology of enlarged lymph-node. Bombay Hospital Journal 42, 1-7.

Singh, J.P., Chaturvedi, N.K. \& Das, A. (1989) Role of fine needle aspiration cytology in the diagnosis of tuberculous lymph adenitis. India Journal of Pathology and Microbiology $32,2$.

Sunpaweravong, P., Sangkhathat, S., Spriplung, H; Chayakul, P (1999) Diagnostic yield of lymphnode aspiration biopsy compared with conventional biopsy Songkla Medical Journal 17, 195-199.

WHO (2000) Acid-fast Direct Smear Microscopy: A Laboratory Training Program. World Health Organization, Geneva.

WHO (2008) World Health Organization: Global Tuberculosis Control, Surveillance, Planning, Financing. WHO Report/HTM/TB/2008.393. Geneva: Switzerland.

Zeer, W., Poliakov, N. \& Abraham, M. (2000) Diagnosis and treatment of cervical tuberculosis lymphadenitis. Journal of Oral Maxillofacial Surgery 58, 477-481. 